CORRECTION

\title{
2010 Annual Meeting Program
}

In the 2010 Annual Meeting Program for the American Academy of Neurology (Neurology ${ }^{\circledR}$ Volume 74, Number 9, Supplement 2, March 2, 2010), the following abstracts were inadvertently omitted.

\section{P01.294}

Tuesday, April 13, 2010

7:30 am-12:00 pm

\section{Regulatory Role of Short Interval Cortical Inhibition During Somatosensory Evoked Motor Cortex Plasticity}

Behzad Elahi, Carolyn Gunraj, Robert Chen, Toronto, ON, Canada

Objective: Paired Associative Stimulation was used to assess the role of inhibitory intra-cortical circuits in motor cortex plasticity. Background: Slow-rate repetitive median nerve stimulation followed by single pulse transcranial magnetic stimulation (TMS) can increase the amplitude of motorevoked potentials (MEP) in the median nerve innervated motor area of small hand muscles at the interstimulus interval of $25 \mathrm{~ms}$. This phenomenon is termed paired associative stimulation (PAS25), and shares a number of physiological aspects of long term potentiation (LTP) in motor cortex. Design/ Methods: Nine healthy subjects were recruited. The study included three different experiments, performed in separate sessions at least one week apart in pseudorandom order. During the process of PAS in order to assess the role of inhibitory intra-cortical circuits in motor cortex plasticity; Median nerve stimulation was conditioned with paired pulse cortical stimulations (CS2-PAS and CS2-PASadj). Results: We found SICI decreased after PAS25 in subjects with stronger inhibition at their baseline and increase in the ICF after PAS25 in subjects with larger facilitation at baseline. Applying SICI together with PAS in CS2-PAS25 condition increased SICI compared to PAS25. SAI was increased in CS2-PAS25adj compared to PAS 25 and CS2-PAS25. MEP measurements during PAS showed similar MEP amplitudes at beginning of stimulation between PAS25 and CS2-PAS25. MEP amplitudes in PAS25 gradually increase after 7 minutes but the MEP amplitudes for CS2-PAS25 remained unchanged. Changes in CS2PAS25adj were increased at two time points after the stimulation but not at the end of the intervention. Conclusions/ Relevance: Our present data show that PAS25 can induce long lasting changes in the GABAergic inhibitory networks responsible for SICI as well as excitatory ICF circuit. Simultaneous stimulation of SICI in conditioned PAS results in increased inhibition in SICI and SAI circuits.

Disclosure: Dr. Elahi has nothing to disclose. Dr. Gunraj has nothing to disclose. Dr. Chen has received personal compensation for activities with Allergan, Inc., Teva Neuroscience, and Medtronic, Inc., as a consultant. Dr. Chen has received research support from Medtronic, Inc.

\section{P02.292 \\ Tuesday, April 13, 2010 \\ 3:00 pm-7:30 pm \\ Cardiopulmonary Fitness is Associated with Cognitive Performance in Patients with Coronary Artery Disease}

Walter Swardfager, Nathan Herrmann, Susan Marzolini, Marilyn Sherman, Alexander Kiss, Prathiba Shammi, Paul Oh, Krista Lanctot, Toronto, ON, Canada

Objective: To investigate the association between cardiopulmonary fitness and cognitive performance in subjects with known coronary artery disease (CAD). Background: Deficits in executive function and memory are common in patients with cardiovascular disease. In medically healthy subjects, poorer cardiopulmonary fitness has been associated with cognitive decline. Design/Methods: Cardiopulmonary fitness was assessed in 81 subjects with CAD entering a cardiac rehabilitation program by measuring peak oxygen uptake (VO2peak) directly in a standardized exercise stress. The fraction of the predicted age and gender norm for VO2peak was computed for each patient. A battery of neuropsychological tests including the Stroop, Trail Making Test B, Digit Symbol Coding, Revised Brief Visuospatial Memory Test, California Verbal Learning Test 2nd Ed. and the Mini Mental Status Exam (MMSE), was administered from which composite Z-scores were computed for tasks involving executive functions and memory. Results: VO2peak was associated with executive function $(\beta=.666, \mathrm{p}<.0005)$, memory $(\beta=.398, \mathrm{p}=.003)$ and MMSE scores $(\beta=.400, \mathrm{p}=$ $.005)$ in multiple linear regression models controlling for potential confounders. The models predicted $36 \%, 16 \%$ and $12 \%$ of the variance in executive function, memory and MMSE scores, respectively. Conclusions/Relevance: Poorer VO2peak is associated with poorer cognition, especially executive function, in subjects with $\mathrm{CAD}$ independent of other cardiac risk factors. Cardiopulmonary fitness may be a key protective factor for cognition in patients with CAD. Study Supported by: The study was supported by The Drummond Foundation, the Physicians' Services Incorporated Foundation, the Heart and Stroke Foundation and the Toronto Rehabilitation Institute.

Disclosure: Dr. Swardfager has nothing to disclose. Dr. Herrmann has received research support from Sonexa Therapeutics Inc. and Lundbeck Canada Inc. Dr. Marzolini has received compensation and/or their research work has been funded, entirely or in part, by a grant to their university. The grant agreement requires that the name of the funding entity and the purpose of the grant may not be disclosed. The funding entity is an other for-profit organization. Dr. Sherman has nothing to disclose. Dr. Kiss has nothing to disclose. Dr. Shammi has nothing to disclose. Dr. Oh has received personal compensation for activities with Pfzer Inc. Dr. Oh has received research support from Pfizer Inc. Dr. Lanctot has received personal compensation for activities with the Medical Outcomes and Research in Economics (MORE) Research Group as an employee. Dr. Lanctot has received research support from Abbott Laboratories, Sonexa Therapeutics Inc., Workers Safety and Insurance Board (WSIB) and Lundbeck Canada. 


\section{Neurology}

\section{Annual Meeting Program}

Neurology 2010;75;839

DOI 10.1212/WNL.0b013e3181f3ed5d

\section{This information is current as of August 30, 2010}

\section{Updated Information \&}

Services

Permissions \& Licensing

\section{Reprints}

including high resolution figures, can be found at: http://n.neurology.org/content/75/9/839.full

Information about reproducing this article in parts (figures,tables) or in its entirety can be found online at:

http://www.neurology.org/about/about_the_journal\#permissions

Information about ordering reprints can be found online:

http://n.neurology.org/subscribers/advertise

Neurology ${ }^{\circledR}$ is the official journal of the American Academy of Neurology. Published continuously since 1951, it is now a weekly with 48 issues per year. Copyright . All rights reserved. Print ISSN: 0028-3878. Online ISSN: 1526-632X.

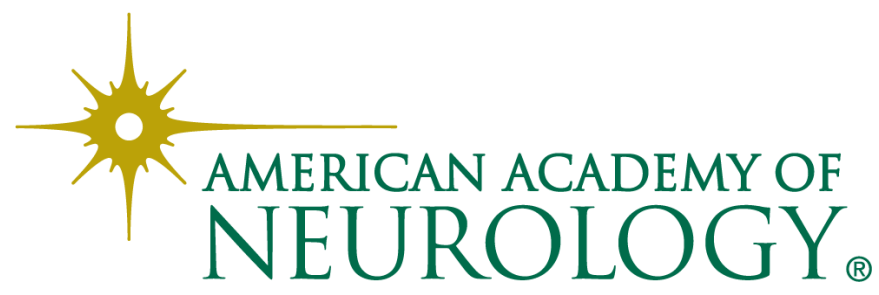

